\title{
Optical education in medicine
}

William Davies, Darren Thomas

William M. Davies, Darren Thomas, "Optical education in medicine," Proc. SPIE 3190, Fifth International Topical Meeting on Education and Training in Optics, (8 December 1997); doi: 10.1117/12.294383

Event: Fifth International Topical Meeting on Education and Training in Optics, 1997, Delft, Netherlands 


\title{
Optical Education in Medicine
}

\author{
William Davies \\ Department of Medical Physics, Swansea NHS Trust Swansea UK, SA2 8QA \\ Darren Thomas \\ Faculty of Applied Engineering and Design \\ Swansea Institute of Higher Education Mount Pleasant Campus Swansea UK
}

\begin{abstract}
A multilevel skills approach has been developed to convey information on optical techniques to a wide range of healthcare staff whose basic technical and scientific education varies considerably. The typical problems encountered in the delivery and assessment of the information are considered in the context of an operational hospital with a range of optical and electrooptical equipment.
\end{abstract}

Keywords Optics, Training, Medicine, CD ROM

\section{OPTICAL EQUIPMENT}

A hospital utilises a range of optical and electrooptical systems that are a mix of "turn key" and experimental equipment. The equipment may be found either in the laboratory or close to the patient bedside with a strong trend towards the latter.

The equipment is broadly grouped into three categories.:

Analytical laboratory equipment such as microscopes, spectroradiometers, florescence analysers and cell counters.

Diagnostic equipment such as ophthalmic assessment units, fibre optic sensors, electro-optic image intensifiers. Also included for training purposes in this group are non optical devices such as phase array ultrasound units that are most easily described by considering the optical principles of diffraction and interference.

Therapeutic equipment such as lasers and other optical radiation sources e.g. . PUVA therapy cabinets.

As the equipment and associated techniques migrate from the laboratory environment towards the patient bedside it places an enhanced need for understanding on a group of staff whose initial technical and scientific education is usually quite limited.

\section{STAFF GROUPS}

In any large organisation, especially a multidisciplinary one as found in a hospital, there is a need to ensure that the various groups receive the correct information to enable them to work effectively and safely.

The need for both the level and detail of training will vary according to the operational needs of the staff concerned.

The following staff groups should be considered

\begin{tabular}{|l|l|l|l|}
\hline Staff Group & Requirements & Technical Skill & Training Need \\
\hline Administration & Low & Low & Level 1 \\
\hline Nursing & High & Low/Medium & Level 1/2 \\
\hline Paramedical & low/High & Low/Medium & Level 1/2 \\
\hline Medical & High & Medium & Level 1/2 \\
\hline Technical & Very High & High & Level 1/2/3 \\
\hline Scientific & Very High & Very High & Level 1/2/3/4 \\
\hline
\end{tabular}


Administrative staff often control the flow of money or other resources to the operational arm of the healthcare facility. They need a basic understanding at a low technical level in order to appreciate the way in which the technology will be utilised and to compare it with alternative methodologies.

Nursing and paramedical staff need to have sufficient training to allow them to operate and, on a day to day basis, maintain the equipment. This is a large staff group, often working on a shift rota basis through 24 hours, and poses considerable practical problems in training delivery.

Medical staff need a thorough understanding of the mechanisms by which the equipment delivers a diagnostic result or therapeutic effect. They particularly need to understand the accuracy and limitations of the equipment as used in their particular clinical setting.

Technical staff involved in the routine maintenance and calibration of equipment need a wide range of technical skills to perform their role. As well as basic electronic skills they need a thorough understanding of the care and handling of optical components.

A small number of scientific staff are involved in the design and development of technical equipment. In order to prosecute their design a formal graduate level education is needed in a range of disciplines including optics. This will include a high level of analytical skill as well as practical or laboratory work.

\section{STRUCTURED EDUCATION}

We have utilised a structured pyramidal approach to the delivery of the educational material. This recognises that the numbers of staff requiring each level of training will also vary roughly in this fashion. All staff groups receive the underlying level of training. Although it might be argued that the scientific staff do not need to understand the full details of the specific service training a good knowledge of this aspect of the process will allow them to consider the ease of serviceability in their design, an aspect far too often overlooked.

LEVEL 4 Theory

LEVEL $3 \quad$ Service detail

LEVEL 2 Operational detail

LEVEL $1 \quad$ Basic principles

As an example of how this has been practically embodied into a training program we have considered how the basics of laser equipment is explained to each group.

Laser Optics 1

At this level little or no understanding of physical principles can be assumed and the information is delivered almost at a public level of need with no analytical reference.

The laser is described as being a beam of light, a little like the beam from a torch except that it is far more collimated and usually all of one "colour". It is sometimes difficult to put over the concept of invisible radiation so general reference is made to colour of the beam. Simple lenses may be introduced to illustrate how the light is brought to a small spot but reference to power or energy density may well not be understood..

At the next level it is necessary to describe how the characteristics of the laser beam may be utilised to produce a range of different effects in tissue. An understanding of power and energy density is necessary together with familiarity with commonly used units. As in this case we are looking at a surgical process we need to discuss the effects of the various parameters associated with laser light such as power/energy density, pulse widths, wavelength on the target tissue.

The service technical staff will gain much from understanding how the laser may be used. It has to be remembered that they will often be the point of contact with the surgeon or other staff and the confidence given by understanding the wider process will be of benefit. A good knowledge of instrumentation and systems is needed together with the ability to understand how to measure and adjust the key parameters such as power/energy output, alignment of the laser system and pulse characteristics. 
Finally we look at the needs of the graduate design engineer and his/her requirement to have a thorough analytical understanding of the optical components of the equipment. This information will usually be taught in a sound academic environment that is able to provide a robust practical experience. The underpinning levels of knowledge will however provide invaluable insight into the operational and practical needs of users of the system and should be embodied into the training at some point.

\section{EDUCATIONAL RESOURCES}

The delivery of all training is usually a compromise between the costs involved, both in time and money and the knowledge uptake of the student. A measure of prior screening of educational background may be feasible in the formal academic environment but is far more difficult in the workplace.

The perception of the need for training is also variable in that enthusiasm for participation or motivation for training may not be present in the individual to the same degree. This greatly influences the use of various resources that may be available for course delivery.

It is normally most efficient to have a direct lecture or tutorial with a person knowledgeable in the subject and allow sufficient opportunity for questions. This is normally quite expensive and additionally needs a good teacher.

Alternative methods such as slide or video material are substantially less expensive, particularly where large numbers of trainees are involved but not so efficient.

A compromise which we have explored is the use of the interactive CD ROM where sets of problems or questions may be interspersed with the information to allow continuous personal or tutor assessment of progress. The design of these programs is expensive but with input from a wider body of expertise they offer consistency and inexpensive reproducibility. Although the playback equipment was expensive trends in multimedia platforms have reduced the costs significantly. A great deal of thought must be undertaken into both the user interface and the presentation of information on the screen. Authoring systems such as icon author $\odot$ and consideration of $\mathrm{HCI}$ ( human computer interaction ) have reduced the development lead time.

\section{EVALUATION \& ASSESSMENT}

One of the most difficult areas of teaching is in the assessment of the knowledge retained by the trainee immediately after the training and at some practical future date. As much of the training we undertake has serious implications for the well-being of the patient it is important to carry out a useful amount of audit into the acquired trainee skills.

There are obviously a number of traditional techniques that can be utilised either singly or more often in some combination. The level of audit has to be consistent with the added responsibility put on the trainee who completes the course. Additionally for many trainees some level of recurring audit is going to be necessary and a simple mechanism for review is required. Some techniques such as end testing are more appropriate to academic courses whilst questionnaires generally are fairly ambivalent.

\section{CONCLUSIONS}

We have tried to approach the task of teaching quite complex optical principles by following a structured technique of training. We have utilised all the current available technologies such as interactive CD ROM and have toiled over the most effective method of assessment that delivers a level of reassurance at the workface. We have attempted to ensure that our teaching material is as animated as possible to increase the level of attendee attention.

\section{ACKNOWLEDGEMENTS}

This work was, in part, sponsored by the EUREKA 643 laser safety program. 